\title{
Alterações do peridomicílio e suas implicações para o controle do Triatoma brasiliensis
}

\author{
Peridomiciliary changes and implications \\ for Triatoma brasiliensis control
}

José Wellington Oliveira-Lima 1

Osvaldo F. Faria Filho 1

João Batista Furtado Vieira 1

Francisco Vieira Gadelha 1

Alfredo M. Oliveira Filho 2

\footnotetext{
1 Fundação Nacional de Saúde do Ceará.

Av. Santos Dumont 1890, Fortaleza, CE

60150-160, Brasil.

2 Núcleo de Pesquisa

de Produtos Naturais,

Universidade Federal

do Rio de Janeiro.

Centro de Ciências da Saúde,

bloco 11, Rio de Janeiro, $R J$

21941-590, Brasil.
}

\begin{abstract}
A total of 9,906 annexes from 1,541 rural dwellings of Boa Viagem County, Ceará, Brazil, infested by Triatoma brasiliensis and Triatoma pseudomaculata were investigated and immediately sprayed with pyrethroid insecticide, followed by revisions at 6,12 , and 18 months. The initial infestation rate of annexes was $4.0 \%$, with predominant infestation in animal shelters (7.0\%). Mean insect density was low, regardless of triatomine species or type of annex. A progressive decrease in the number of initial annexes was observed (66\% of remaining annexes), mainly those classified as "piles of materials". Only 3\% of the annexes were modified by the population. New constructed annexes were important as new foci of infestation. Some 25\% were infested at the end of observation period, significantly more than the "old" annexes (4.0\%), a difference attributed to insecticide spraying at the beginning of the intervention. Reinfestation occurred slowly and was more frequent in animal shelters No differences were observed between traditional pyrethroid and slow-release organophosphate formulations. Selective spraying of "new" annexes is recommended.
\end{abstract}

Key words Chagas Disease; Triatominae; Triatoma brasiliensis; Vector Control; Prevention and Control

Resumo Em Boa Viagem, Ceará, foram investigados e rociados 9.906 anexos dos peridomicílios de 1.541 vivendas rurais em área infestada por Triatoma brasiliensis e/ou Triatoma pseudomaculata com revisões aos 6, 12 e 18 meses. Observou-se um decréscimo do número de anexos nos 18 meses, mais intenso para anexos tipo "pilhas de materiais". De modo geral, a população só modificou 3\% dos anexos, principalmente os abrigos de animais (6\%). A construção de anexos novos foi importante como causa de novas infestações, significativamente mais presentes nestes (25\%) que naqueles que existiam desde o início da observação (4\%), provavelmente por conta do expurgo inicialmente realizado. A reinfestação ocorreu lentamente, sendo mais freqüente nos abrigos de animais $(7,0 \%)$ que em pilhas $(4,4 \%)$ e em cercas e cobertas $(<1 \%)$. A densidade da reinfestação sempre foi baixa, independente da espécie do triatomíneo ou do tipo do anexo. Em um estudo colateral, um ensaio pareado com a borrifação inicial de piretróide versus composto organofosforado em matriz de lenta liberação não mostrou diferenças de infestação nem de densidade triatomínica a final do experimento. Sugere-se a borrifação seletiva de anexos novos tão logo sejam construídos, em áreas semelhantes à deste estudo.

Palavras-chave Doença de Chagas; Triatominae; Triatoma brasiliensis; Controle de Vetores; Prevenção e Controle 


\section{Introdução}

A doença de Chagas é endêmica nos Estados do Nordeste do Brasil (Camargo et al., 1984). Nessa região, o Triatoma brasiliensis e o Triatoma pseudomaculata são consideradas as principais espécies vetoras pelo fato de serem as espécies prevalentes no ambiente domiciliar (Forattini, 1980). O espaço ecológico ocupado por essas duas espécies não se restringe ao ambiente domiciliar (Alencar et al., 1977a), podendo também ser estendido aos ambientes modificados que circundam as moradias humanas (Alencar \& Bezerra, 1977) e ao ambiente silvestre (Alencar et al., 1977b). Acredita-se que essa valência ecológica seja o principal obstáculo ao controle efetivo destas duas espécies vetoras através da aplicação intradomiciliar de inseticidas. Diferentemente do que ocorreu com o Triatoma infestans, um vetor que tinha sua distribuição restrita ao ambiente domiciliar (Forattini, 1980), sua eliminação não permitia a reinfestação pela migração de especimens a partir do ambiente silvestre (Silveira, 1985; Silveira \& Rezende, 1994).

Acredita-se que, para o controle químico do T. brasiliensis e do T. pseudomaculata no peridomicílio, é necessário um inseticida que apresente uma ação residual longa nas condições existentes nesse ambiente. Embora alguns piretróides sintéticos apresentem uma ação residual considerável no ambiente intradomiciliar (Pinchin et al., 1980, 1981, 1982, 1984), o mesmo não ocorre no ambiente peridomiciliar (Oliveira Filho, 1988, 1989). Entretanto, mesmo que tal inseticida exista, um outro fator, independente do poder residual do inseticida e com capacidade de comprometer qualquer estratégia de controle químico no peridomicílio, é a renovação desse ambiente peridomiciliar. Com a estratégia usada atualmente pelo Programa de Controle da Doença de Chagas (PCDCh) da Fundação Nacional de Saúde, os domicílios das áreas endêmicas são visitados a cada a um ou dois anos, com a conseqüente identificação e borrifação daqueles encontrados infestados. Destarte, um anexo não permanente do peridomicílio, que tenha surgido depois da visita do Programa, ficará desprotegido pelo inseticida até a próxima visita, se é que ele venha a permanecer no peridomicílio até essa próxima visita. Anexos não permanentes são: pilhas de madeira, de telha, de tijolos; abrigos de animais, etc. Nessas condições, se essa renovação do peridomicílio implica na presença constante de potenciais abrigos de triatomíneos não protegidos pelo inseticida, a efetividade de um inseticida poderá ser comprometida, qualquer que seja a duração de seu efeito residual.
Neste trabalho, apresentamos os resultados de um estudo da dinâmica do peridomicílio no Ceará, Brasil, realizado com o objetivo de se medir o grau e a velocidade com que os elementos que o compõem são eliminados, modificados e renovados, em uma área endêmica para T. brasiliensis e T. pseudomaculata.

\section{Material e métodos}

\section{Área de estudo}

A área de estudo situa-se no Município de Boa Viagem, no Estado do Ceará. Essa área foi selecionada por estar entre os municípios de fácil acesso, a partir de Fortaleza, que apresentava maior percentual de domicílios infestados por triatomíneos e que se encontrava há mais tempo sem receber tratamento com inseticida, de acordo com os registros do PCDCh, da Fundação Nacional de Saúde, no Ceará. Foram selecionadas 68 localidades contíguas, que incluíam 1.541 domicílios. O Município de Boa Viagem localiza-se a uma latitude de 5o $07^{\prime} 39^{\prime}$ e longitude 39o 43" 56". Apresenta temperatura que oscila entre $25^{\circ} \mathrm{C}$ e $35^{\circ} \mathrm{C}$ e uma precipitação pluviométrica anual média de $704 \mathrm{mmHg}$. A área é coberta por floresta caducifólia espinhosa e subcaducifólia tropical pluvial, assim como caatinga arbustiva densa (IPLANCE, 1998).

\section{Classificação dos anexos}

Qualquer coisa existente no peridomicílio com capacidade para abrigar triatomíneos foi considerada "anexo". "Peridomicílio" foi definido com sendo a área existente ao redor do domicílio, num raio não superior a cem metros. Os anexos foram caracterizados de acordo com a presença ou não de cobertura, se usados como abrigo de animais ou se constituíam simplesmente de pilhas (amontoados) de tijolos, de telhas ou de madeira. Cercas também foram classificadas como um tipo específico de anexo. Foi elaborado um detalhado mapa pictórico do peridomicílio, onde era assinalada a presença de todos os anexos com um número identificador e, numa anotação complementar, eram descritas em detalhes as suas características.

\section{Acompanhamento dos anexos}

Os anexos foram examinados em quatro momentos diferentes. Em um primeiro momento (avaliação inicial), e depois de 6, 12 e 18 meses (avaliações de seguimento). Os anexos presentes no peridomicílio na avaliação inicial foram 
chamados de "anexos velhos". "Anexos modificados" foram os anexos velhos nos quais se observou qualquer alteração, seja por supressão ou por acréscimo de parte, nas avaliações de seguimento de 6, 12 e 18 meses. "Anexos novos" foram aqueles observados nas avaliações de seguimento e que não estavam presentes na avaliação inicial. Por último, registramos os "anexos demolidos", que foram os anexos velhos que não estavam mais presentes no peridomicílio nas avaliações de seguimento.

\section{Amostragem de triatomíneos}

Todos os espaços existentes nos anexos do peridomicílio, com capacidade de abrigar triatomíneos, foram examinados com o auxílio de uma lanterna, durante o tempo que fosse necessário para se proceder a um exame exaustivo. Os triatomíneos encontrados eram recolhidos e levados ao laboratório para classificação, segundo a chave de Lent \& Wygodzinsky (1979).

\section{Análise dos dados}

Para cada tipo de anexo, calculamos o percentual acumulado da condição de ser velho na avaliação inicial e da de ser velho, modificado ou novo nas avaliações de 6, 12 e 18 meses. Também calculamos a prevalência de anexos infestados por triatomíneos, segundo o tipo de anexo. Para verificarmos se existiam diferenças entre as proporções observadas, calculamos o Qui-Quadrado e a probabilidade (valor-p) de as proporções serem semelhantes.

\section{Resultados}

Na Tabela 1, apresentamos os anexos que ao longo dos 18 meses de observação não sofreram qualquer modificação e onde, portanto, poder-se-ia esperar que um inseticida, dotado de um poder residual com essa duração, seria eficaz. Observamos que, após 18 meses, aproximadamente $66 \%$ dos anexos permanecem sem qualquer alteração. Dos abrigos de animais, permaneciam $61 \%$; das áreas cobertas, $82 \%$; das cercas, $80 \%$; das pilhas, $39 \%$. Pilhas e abrigos de animais são os mais prováveis de desaparecerem ou de serem modificados, enquanto área coberta e cercas são as menos prováveis.

Na Tabela 2, apresentamos a evolução da população supra de anexos modificados, onde esperamos que um inseticida tenha sua eficácia diminuída mesmo que seu poder residual persista aos 18 meses. Ao final dos 18 meses, 3\% dos anexos presentes no peridomicílio haviam sido modificados, sendo que houve modificação em $6 \%$ dos abrigos de animais, em $2 \%$ das áreas cobertas e em menos de $1 \%$ das cercas e pilhas. Os dados sugerem que as modificações de anexos não devem ser um fator importante que comprometa o poder residual de um inseticida.

$\mathrm{Na}$ Tabela 3, mostramos a incidência de anexos novos ao longo dos 18 meses de observação. Os anexos novos são os mais suscetíveis de serem colonizados por triatomíneos, uma vez que não foram tratados pelo inseticida, pois não estavam presentes no peridomicílio no momento da borrifação. Ao final do período de observação, 25\% dos anexos presentes no peridomicílio eram novos. A maior incidência observada foi de pilhas (46\%), seguindo-se de abrigos de animais (28\%), áreas cobertas (18\%) e cercas (14\%). Concluímos que o estabelecimento de novos anexos no peridomicílio é uma ocorrência muito freqüente e que esse fator pode aumentar consideravelmente o risco de colonização do peridomicílio por triatomíneos.

Medimos o risco de colonização dos diversos tipos de anexos pelo T. brasiliensis e/ou $T$. pseudomaculata através da freqüência da infestação (Tabela 4). Os anexos mais freqüentemente colonizados foram os abrigos de animais $(7,0 \%)$ e as pilhas $(4,4 \%)$. Áreas cobertas e cercas apresentaram uma prevalência menor que $1 \%$. Os triatomíneos apresentam uma preferência significativamente maior por abrigos de animais e pilhas (amontoados).

Alternativamente, medimos a suscetibilidade à colonização dos diversos tipos de anexos pelo T. brasiliensis e/ou T. pseudomaculata através da intensidade da infestação (Tabela 5). A grande maioria dos anexos infestados abrigavam de um a quatro triatomíneos, não existindo diferenças significativas dos percentuais de cada tipo de anexo classificado de acordo com o número de especimens de triatomíneos encontrados. Os dados sugerem que o número de triatomíneos encontrados num determinado anexo não depende do tipo do mesmo.

\section{Discussão}

No Brasil, entre as doenças transmitidas por vetores, a doença de Chagas é considerada uma das mais importantes como problema de saúde pública, seja por sua elevada prevalência e extensa distribuição geográfica em algumas regiões do país, seja pela alta letalidade de formas clínicas da doença (Schmunis, 1997). Até o presente, não existe nenhum recurso terapêutico eficaz contra as complicações crônicas da doença, notadamente o megacolo, o megaesô- 
Anexos que permaneceram íntegros ao longo de 18 meses, numa área endêmica para Triatoma brasiliensis e Triatoma pseudomaculata, no Município de Boa Viagem, Ceará.

\begin{tabular}{|c|c|c|c|c|c|c|c|c|}
\hline \multirow[t]{3}{*}{ Tipos de anexo } & \multicolumn{8}{|c|}{ Números e percentagens acumuladas de anexos velhos } \\
\hline & \multicolumn{2}{|c|}{ Levantamento inicial } & \multicolumn{2}{|c|}{6 meses } & \multicolumn{2}{|c|}{12 meses } & \multicolumn{2}{|c|}{18 meses } \\
\hline & $\mathrm{n}$ & $\%$ & $\mathrm{n}$ & $\%$ & $\mathrm{n}$ & $\%$ & $\mathrm{n}$ & $\%$ \\
\hline Abrigos de animais & 4.580 & 46 & 3.575 & 78 & 3.131 & 68 & 2.776 & 61 \\
\hline Áreas cobertas & 590 & 6 & 526 & 89 & 501 & 85 & 481 & 82 \\
\hline Cercas & 3.435 & 35 & 3.128 & 91 & 2.921 & 85 & 2.758 & 80 \\
\hline Pilhas & 1.301 & 13 & 763 & 59 & 620 & 48 & 502 & 39 \\
\hline Total & 9.906 & 100 & 7.992 & 81 & 7.173 & 72 & 6.517 & 66 \\
\hline
\end{tabular}

Tabela 2

Anexos modificados ao longo de 18 meses, numa área endêmica para Triatoma brasiliensis e Triatoma pseudomaculata, no Município de Boa Viagem, Ceará.

\begin{tabular}{|c|c|c|c|c|c|c|c|}
\hline \multirow[t]{3}{*}{ Tipos de anexo } & \multicolumn{7}{|c|}{ Números e percentagens acumuladas de anexos modificados } \\
\hline & \multirow[t]{2}{*}{ Levantamento inicial } & \multicolumn{2}{|c|}{6 meses } & \multicolumn{2}{|c|}{12 meses } & \multicolumn{2}{|c|}{18 meses } \\
\hline & & $\mathrm{n}$ & $\%$ & $\mathrm{n}$ & $\%$ & $\mathrm{n}$ & $\%$ \\
\hline Abrigos de animais & 4.580 & 232 & 5 & 252 & 6 & 265 & 6 \\
\hline Áreas cobertas & 590 & 9 & 2 & 11 & 2 & 14 & 2 \\
\hline Cercas & 3.435 & 11 & 0 & 16 & 0 & 23 & 1 \\
\hline Pilhas & 1.301 & 3 & 0 & 2 & 0 & 1 & 0 \\
\hline Total & 9.906 & 255 & 3 & 281 & 3 & 303 & 3 \\
\hline
\end{tabular}

Tabela 3

Anexos novos que apareceram ao longo de 18 meses, em uma área endêmica para Triatoma brasiliensis e Triatoma pseudomaculata, no Município de Boa Viagem, Ceará.

\begin{tabular}{|c|c|c|c|c|c|c|c|}
\hline \multirow[t]{3}{*}{ Tipos de anexo } & \multicolumn{7}{|c|}{ Números e percentagens acumuladas de anexos novos } \\
\hline & \multirow[t]{2}{*}{ Levantamento inicial } & \multicolumn{2}{|c|}{6 meses } & \multicolumn{2}{|c|}{12 meses } & \multicolumn{2}{|c|}{18 meses } \\
\hline & & $\mathrm{n}$ & $\%$ & $\mathrm{n}$ & $\%$ & $\mathrm{n}$ & $\%$ \\
\hline Abrigos de animais & 4.580 & 705 & 15 & 1.146 & 25 & 1.278 & 28 \\
\hline Áreas cobertas & 590 & 66 & 11 & 98 & 17 & 109 & 18 \\
\hline Cercas & 3.435 & 282 & 8 & 433 & 13 & 484 & 14 \\
\hline Pilhas & 1.301 & 406 & 31 & 553 & 43 & 600 & 46 \\
\hline Total & 9.906 & 1.452 & 15 & 2.230 & 23 & 2.471 & 25 \\
\hline
\end{tabular}


Tabela 4

Prevalência de infestação de anexos por Triatoma brasiliensis e/ou Triatoma pseudomaculata, no Município de Boa Viagem, Ceará.

\begin{tabular}{lcrc}
\hline Tipos de anexo & Existentes no levantamento inicial & Número e percentual de anexos infestados \\
$\%$
\end{tabular}

¥ Comparação das proporções: $\chi^{2}=225$ (com 2 g.l.); $p=0,000$

Tabela 5

Número de Triatoma brasiliensis e Triatoma pseudomaculata amostrados em anexos de domicílios, no Município de Boa Viagem, Ceará

\begin{tabular}{|c|c|c|c|c|c|}
\hline \multirow[t]{3}{*}{ Tipos de anexo } & \multirow[t]{3}{*}{ Número de anexos coletados infestados } & \multicolumn{4}{|c|}{$\begin{array}{l}\text { Anexos infestados, segundo } \\
\text { o número de triatomíneos }\end{array}$} \\
\hline & & \multicolumn{2}{|c|}{ 1-4 triatomíneos } & \multicolumn{2}{|c|}{$5-13$ triatomíneos } \\
\hline & & $\mathrm{n}$ & $\%$ & $n$ & $\%$ \\
\hline Abrigos de animais & 320 & 259 & 81 & 61 & 19 \\
\hline Pilhas & 57 & 41 & 72 & 16 & 28 \\
\hline Áreas cobertas e cercas & 24 & 21 & 88 & 3 & 12 \\
\hline Total & 401 & 319 & 80 & 80 & 20 \\
\hline
\end{tabular}

fago e a cardiopatia chagásica, assim como não existe nenhuma vacina que evite a infecção ou a evolução da infecção da forma indeterminada para as formas crônicas nos indivíduos infectados (WHO, 1991). Desta forma, a única possibilidade de evitar o aparecimento das formas clínicas debilitantes e letais da doença de Chagas reside no controle da transmissão, seja da transmissão vetorial ou da transmissão por transfusão de sangue.

Ecologicamente, a doença de Chagas é uma doença típica de ambientes rurais, onde o Trypanosoma cruzi circula entre animais silvestres, peridomésticos, domésticos e o homem, transmitido por diversas espécies de vetores, triatomíneos hematófagos. Quanto mais imbricada for a residência humana nesse ambiente, maior a chance de o homem fazer parte do ciclo de transmissão. Estudos têm demonstrado que as moradias de baixo padrão de construção, que apresentam muitos locais adequados para abrigar triatomíneos, são as que oferecem maior risco de infecção para seus moradores (Mott et al., 1978).
Entre outras, duas estratégias têm sido propostas para o controle da densidade dos triatomíneos transmissores da doença de Chagas: a melhoria habitacional (Schofield \& Marsden, 1982) e o controle químico (WHO, 1991). A melhoria habitacional tem sido aplicada em áreas restritas do Brasil, e, até o momento, nenhuma avaliação sistemática foi realizada concernente à eficácia desta estratégia. Por outro lado, o controle químico de populações de triatomíneos tem sido extensivamente usado nas áreas endêmicas do Brasil (Silveira, 1985). No Brasil, na área de distribuição do Triatoma infestans, os excelentes resultados de anos seguidos de rociado das habitações humanas, inicialmente com inseticidas organoclorados e, depois, com inseticidas do grupo piretróide, levaram o Ministério da Saúde do Brasil a declarar a interrupção da transmissão vetorial da doença de Chagas em extensas áreas do Sul e Centro-Sul do País (Silveira \& Rezende, 1994). Esses resultados abriram uma perspectiva de que seria possível também interromper a transmissão vetorial em outras regiões do país, como, por 
exemplo, o Nordeste Brasileiro, área de distribuição do T. brasiliensis e T. pseudomaculata.

No Ceará, o controle químico da doença de Chagas teve início de forma sistemática em 1975 , no vale do Jaguaribe, naquele momento a principal área endêmica do estado, com uma grande expansão para outras áreas endêmicas do estado, em 1983. Atualmente, cerca de $90 \%$ da área potencialmente endêmica da doença de Chagas do Ceará são cobertos pelo programa. No Vale do Jaguaribe, área endêmica do $T$. brasiliensis por excelência, conseguiu-se, após 16 anos ininterruptos de controle, uma redução significativa do percentual de casas infestadas, assim como do percentual de triatomíneos naturalmente infectados (Oliveira-Lima et al., 1992). Apesar dos excelentes resultados observados, ao final de 16 anos de trabalho ainda existia um resíduo importante de domicílios infestados.

Resultados como esses sustentaram a hipótese de que seria muito difícil obter um impacto expressivo mediante o controle químico de espécies de triatomíneos que não colonizam exclusivamente o intradomicílio, pois, uma vez cessado o efeito residual do inseticida aplicado no intradomicílio, o mesmo seria recolonizado por especimens porventura existentes no peridomicílio e/ou no ambiente silvestre. Em outras palavras, controlar o T. brasiliensis e o T. pseudomaculata implicava controlar populações de triatomíneos no peridomicílio. Nessas circunstâncias, seria pertinente a busca de um inseticida novo ou de uma nova formulação de inseticidas já existentes que apresentasse um efeito residual prolongado no peridomicílio, uma vez que os inseticidas e/ou formulações existentes no mercado apresentavam um efeito residual de curta duração (Oliveira Filho, 1996, 1997). Essa lacuna foi preenchida com o aparecimento do Durathion, que é uma nova formulação do Malathion, desenvolvida pelo Núcleo de Pesquisas de Produtos Naturais da Universidade Federal do Rio de Janeiro (Oliveira Filho, 1988). Ensaios preliminares de campo mostraram que as superfícies borrifadas com essa formulação de lenta liberação de Fenitrothion (ação prolongada) ainda apresentavam ação inseticida 18 meses após a aplicação (Oliveira Filho et al., 1996).

Numa área de distribuição do T. brasiliensis e T. pseudomaculata, foi implementado um grande ensaio de campo para comparar, no peridomicílio, o efeito residual do Durathion com o da Deltametrina. Doze meses após o rociado, os percentuais de peridomicílios infestados foram semelhantes em ambas as áreas (Faria Filho et al., 1998). Estes resultados não estavam de acordo com aqueles reportados (Oliveira Filho et al., 1996). Na busca de uma explicação para estes resultados, levantamos a hipótese de que na nossa área de estudo, depois de 12 meses, o Durathion não exerceria seu poder residual porque os anexos borrifados teriam sido substituídos ou modificados.

Neste estudo, observamos que, depois de 18 meses, $66 \%$ dos diversos tipos de anexos que haviam sido borrifados ainda permaneciam no peridomicílio (anexos velhos). Se o inseticida usado tiver um efeito residual de pelo menos 18 meses, presume-se que o inseticida remanescente nesses anexos velhos ainda tenha a capacidade de intoxicar os triatomíneos que porventura venham a se alojar neles. Talvez, mais importante que a permanência dos anexos velhos que conservam o poder residual do inseticida seja o aparecimento de anexos novos, sem essa proteção, onde os triatomíneos podem viver e reproduzir-se. Depois de $18 \mathrm{me}-$ ses, $25 \%$ dos anexos presentes no peridomicílio eram anexos novos, ou anexos que apareceram depois do rociado e que não apresentavam nenhum poder inseticida.

Adicionalmente, o impacto da renovação dos anexos na recolonização do peridomicílio depende não apenas da velocidade da renovação, mas também da preferência dos triatomíneos por cada tipo de anexo. Observamos que os triatomíneos colonizam mais freqüentemente os abrigos de animais e as pilhas do que as áreas cobertas e as cercas. Esses abrigos preferidos pelo triatomíneos são exatamente os tipos de anexos que se renovam mais rapidamente. Acreditamos que os anexos modificados tenham uma importância muito menor que os anexos novos, pois neles é de se esperar que algum poder inseticida residual permaneça. De qualquer maneira, no caso particular deste estudo, os anexos modificados constituíram apenas $3 \%$ dos anexos presentes no peridomicílio ao final de 18 meses de observação.

Concluímos que a duração dos ciclos de aplicação de inseticidas para controlar populações de triatomíneos no peridomicílio deve levar em conta não somente a duração do efeito residual do inseticida, mas também a renovação dos anexos do peridomicílio. Deste modo, mesmo com o uso de uma formulação de longo efeito residual no peridomicílio, o Programa de Controle da Doença de Chagas deveria modificar sua estratégia de trabalho, de forma que os domicílios não sejam borrifados apenas ciclicamente, porém sempre que apareça um novo anexo infestado. Este deveria então ser, de imediato, tratado. Tal procedimento só será possível adotando-se uma estratégia de controle mais horizontal do que aquela que é praticada atualmente. 


\section{Referências}

ALENCAR, J. E.; ALMEIDA, Y. M.; FREITAS, L. M. \& SANTOS, A. R., 1977a. Estudos sobre a epidemiologia da doença de Chagas do Ceará - Brasil. VI Estudos de uma micro-região de Russas. Revista da Sociedade Brasileira de Medicina Tropical, 11: 5-23.

ALENCAR, J. E.; CUNHA, R. V.; ARAÚJO, A. G. S. C. \& SOBREIRA, A., 1977b. Epidemiologia da doença de Chagas do Ceará. X - Hábitos alimentares dos vetores. Revista da Sociedade Brasileira de Medicina Tropical, 11:5-23.

ALENCAR, J. E. \& BEZERRA, F., 1977. Epidemiologia da doença de Chagas do Ceará. IX - Estudo de vetores numa área endêmica (Morada Nova). Revista da Sociedade Brasileira de Medicina Tropical, 11:25-44.

CAMARGO, M. E.; SILVA, G. R.; CASTILHO, E. A. \& SILVEIRA, A. C., 1984. Inquérito sorológico da prevalência da doença de Chagas no Brasil, 1975/ 1980. Revista do Instituto de Medicina Tropical de São Paulo, 26:192-204.

FARIA FILHO, O. F.; GADELHA, F. V.; CARNEIRO, F. C. F; LIMA, J. N. O. \& OLIVEIRA FILHO, A. M., 1998. Ensaio para avaliação do efeito residual do Duration M no controle do Triatoma brasiliensis e $T$. pseudomaculata. Revista da Sociedade Brasileira de Medicina Tropical, 31:58.

FORATTINI, O. P., 1980. Biogeografia, origem e distribuição da domiciliação de triatomíneos no Brasil. Revista de Saúde Pública, 14:265-99.

IPLANCE (Fundação Instituto de Planejamento do Ceará), 1998. Perfil Básico Municipal. Fortaleza: Edições IPLANCE.

LENT, H. \& WYGODZINSKY, P., 1979. Revision of the triatominae (Hemiptera, Reduviidae), and their significance as vectors of Chagas' Disease. Bulletin of the American Museum of Natural History, 163:3.

MOTT, K. E.; MUNIZ, T. M.; LEHMAN, J. S.; HOFF, R.; MORROW, R. H.; OLIVEIRA, T. S.; SHERLOCK, I. \& DRAPER, C. C., 1978. House construction, triatominae distribution, and household distribution of seroreactivity to Trypanosoma cruzi in a rural community in Northeast Brazil. American Journal of Tropical Medicine and Hygiene, 7:11161122.

OLIVEIRA FILHO, A. M., 1988. Development of insecticide formulations and determination of dosages and application schedules to fit specific situations. Revista Argentina de Microbiología, 20:3948.

OLIVEIRA FILHO, A. M., 1989. New alternatives for the control of triatomines in peridomestic buildings. Revista da Sociedade Brasileira de Medicina Tropical, 22:53-57.

OLIVEIRA FILHO, A. M., 1996. Recent advances in the use of slow-release insecticide formulations against triatomines. Revista da Sociedade Brasileira de Medicina Tropical, 28:74-78.

OLIVEIRA FILHO, A. M., 1997. Uso de nuevas herramientas para el control de triatominos en diferentes situaciones entomológicas en el continente americano. Revista da Sociedade Brasileira de Medicina Tropical, 30:41-46.
OLIVEIRA FILHO, A. M.; MELO, M. T. V. \& SANTOS, C. E., 1996. The chemical control of triatomines in north-eastern Brazil - Results of a two years large scale field study. In: 14th International Congress for Tropical Medicine and Malaria, Proceedings, p. 85, Nagasaki: Japanese Society of Tropical Medicine.

OLIVEIRA-LIMA, J. W.; FARIA FILHO, O. F. \& TIMBÓ, M. J. M., 1992. Controle de triatomíneos numa área endêmica de doença de Chagas no Ceará Dezesseis anos de trabalho. In: 2o Congresso Brasileiro de Epidemiologia, Resumos, p. 89, Belo Horizonte: Associação Brasileira de Pós-Graduação em Saúde Coletiva - ABRASCO.

PINCHIN, R.; OLIVEIRA FILHO, A. M.; FANARA, D. M. \& GILBERT, B., 1980. A Preliminary Field Trial of Decamethrin (OMS 1998) for the Control of Triatoma infestans. World Health Organization document VBC/80.797. Geneva: World Health Organization.

PINCHIN, R.; OLIVEIRA FILHO, A. M. \& GILBERT, B., 1981. Field trial of Permethrin for the control of Triatoma infestans. Bulletin of the Pan American Health Organization, 15:370-376.

PINCHIN, R.; FANARA, D. M. \& OLIVEIRA FILHO, A. M., 1982. A Village Scale Trial Of PirimiphosMethyl (OMS 1424) Wettable Powder and Slow Release Formulations, in Comparison with HCH for the Control of Triatoma Infestans in Brazil. World Health Organization document VBC/82.840. Geneva: World Health Organization.

PINCHIN, R.; FANARA, D. M. \& OLIVEIRA FILHO, A. M., 1984. A village scale trial of Bendiocarb (OMS 1394) for the control of the Chagas' disease vector Triatoma infestans in Brazil. Insect Science and its Application, 5:121-126.

SCHMUNIS, G. A., 1997. Tripanosomíase americana: Seu impacto nas Américas e perspectivas de controle. In: Clínica e Terapêutica da Doença de Chagas (J. C. P. Dias \& J. R. Coura, org.), pp. 11-23, Rio de Janeiro: Editora Fiocruz.

SCHOFIELD, C. J. \& MARSDEN, P. D., 1982. The effect of wall plaster on a domestic population of Triatoma infestans. Bulletin of the PanAmerican Health Organization, 16:356-359.

SILVEIRA, A. C., 1985. O programa de controle da doença de Chagas no Brasil. Annales de la Societé Belge de Medecine Tropicale, 65:137-148.

SILVEIRA, A. C. \& REZENDE, D. F., 1994. Epidemiologia e controle da transmissão vetorial da doença de Chagas no Brasil. Revista da Sociedade Brasileira de Medicina Tropical, 27:11-22.

WHO (World Health Organization), 1991. Control of Chagas Disease. WHO Technical Report Series 811. Geneva: WHO. 